\title{
Mass and Orbit Constraints of the Gamma-ray Binary LS 5039
}

\author{
T. Szalai ${ }^{1}$, G. E. Sarty ${ }^{2,3}$, L. L. Kiss ${ }^{4}$, J. M. Matthews ${ }^{5}$, J. Vinkó ${ }^{1}$, \\ and C. Kiss ${ }^{4}$ \\ ${ }^{1}$ Department of Optics and Quantum Electronics, University of Szeged, \\ Dóm tér 9., Szeged H-6720, Hungary; email: szaszi@titan.physx.u-szeged.hu \\ ${ }^{2}$ Royal Astronomical Society of Canada, Saskatoon Centre, \\ P.O. Box 317, RPO University, Saskatoon, SK S7N 4J8, Canada \\ ${ }^{3}$ Department of Physics and Engineering Physics, University of Saskatchewan, \\ Saskatoon, SK S7N 5E2, Canada \\ ${ }^{4}$ Konkoly Observatory of the Hungarian Academy of Sciences, \\ H-1525 Budapest, P.O. Box 67, Hungary \\ ${ }^{5}$ Department of Physics and Astronomy, University of British Columbia, \\ 6224 Agricultural Road, Vancouver, BC V6T 1Z1, Canada
}

\begin{abstract}
We present the results of space-based photometric and ground-based spectroscopic observing campaigns on the $\gamma$-ray binary LS 5039. The new orbital and physical parameters of the system are similar to former results, except we found a lower eccentricity. Our MOST-data show that any broad-band optical photometric variability at the orbital period is below the 2 mmag level. Light curve simulations support the lower value of eccentricity and imply that the mass of the compact object is higher than $1.8 \mathrm{M}_{\odot}$.
\end{abstract}

Keywords. binaries: spectroscopic, stars: individual (LS 5039)

\section{Introduction}

LS 5039, the enigmatic high-mass X-ray binary has been intensively observed at various wavelengths in the past years (see Sarty et al. 2011, hereafter S11 for a review). Paredes et al. (2000) identified relativistic radio jets and also a very high energy (VHE) gammaray source at the coordinates of the system; therefore LS 5039 became one of a handful of known gamma-ray binaries. There are several open issues about the system (see S11), but the major question is whether the secondary component orbiting around the $\mathrm{O} 6.5 \mathrm{~V}((\mathrm{f}))$ star is a black hole or a non-accreting young pulsar.

Hereinafter, we show the results of our spectroscopic and photometric analysis concerning mass and orbit constraints of LS 5039 (see the details in S11).

\section{Analysis and parameter determination}

Spectroscopic observations were carried out in 2009 with the Echelle spectrograph mounted at ANU 2.3m Telescope (SSO, Australia), and in 2011 using FEROS (Kaufer et al. 1999) at MPG/ESO-2.2m telescope at La Silla, Chile. Covering $~ 40$ hours with nearly uniform sampling of the whole orbit between $3900-6750 \AA$ with a resolving power $\lambda / \Delta \lambda \approx 23,000$ at $\mathrm{H} \alpha$, it is the highest resolution, homogeneous spectral dataset ever obtained for LS 5039 .

Radial velocities (RV) of HI and HeI lines show a systematic blueshift with respect to the RVs of HeII lines, therefore, only the latter ones were used to fit eclipsing binary 

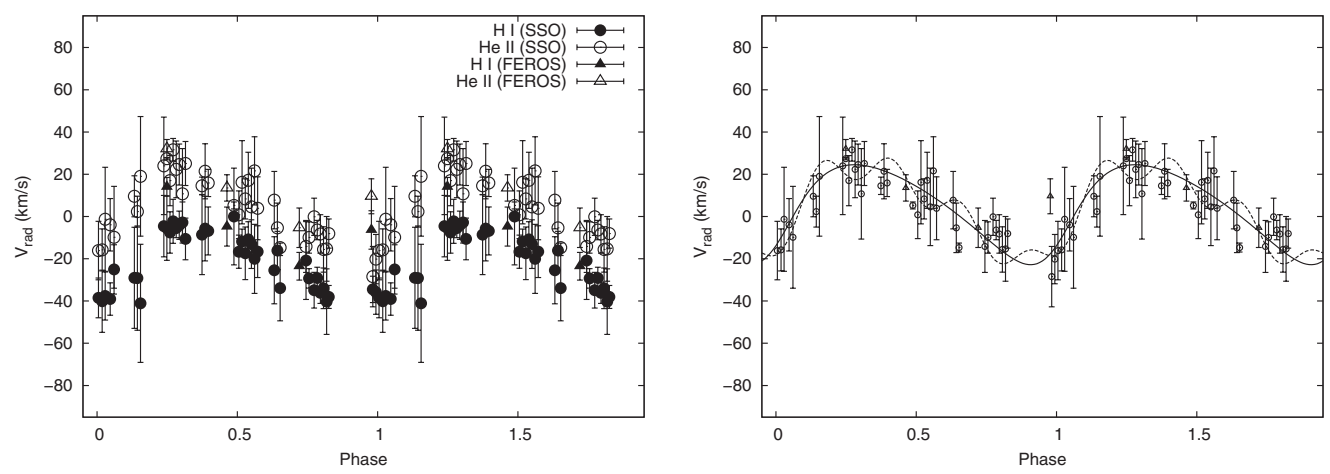

Figure 1. Left: Radial velocities based on $\mathrm{H}$ Balmer and HeII lines. Right: The best-fitting curve to radial velocities of HeII lines (solid line), also with the assumed pulsation of the $\mathrm{O}$ star (dotted line).

models with the Wilson-Devinney (WD) code (Wilson \& van Hamme 2003). We do not see signs of non-radial pulsations in our data in contrast to the results reported by Casares et al. (2010), see Fig. 1.

Our data were analyzed as described in S11, with the addition of the FEROS data. Orbital parameters are close to previous solutions (Casares et al. 2005, Aragona et al. $2009)$, but we found the orbital eccentricity $(e=0.24 \pm 0.08)$ to be definitely lower than determined previously.

Photometric data, obtained with MOST satellite in July of 2009, indicate a variability at the level of 2 mmag. Our light curve (LC) simulations show that the LC amplitude decreases with increasing total mass or decreasing eccentricity. Comparison of data and models suggests that primary mass is at the higher end of estimates based on its spectral type $\left(\sim 26 \mathrm{M}_{\odot}\right)$, from which we get a mass for the compact star of at least $1.8 \mathrm{M}_{\odot}$.

\section{Conclusions}

We carried out a detailed spectroscopic and photometric analysis to get mass and orbit constraints for the $\gamma$-ray binary LS 5039. The new system parameters are close to the previously determined ones, except that we found a lower eccentricity. LC simulations support this result, and imply that the compact object may be a low mass black hole but do not fully exclude that it may be a neutron star.

\section{Acknowledgements}

This work has been supported by the Australian Research Council, the University of Sydney, the Hungarian OTKA Grant K76816, and the "Lendület" Young Researchers' Program of the Hungarian Academy of Sciences.

\section{References}

Aragona, C., McSwain, M. V., Grundstrom, E. D., et al., 2009, ApJ, 698, 514

Casares, J., Ribó, M., Ribas, I., et al., 2005, MNRAS, 364, 899

Casares, J., Corral-Santana, J. M., Herrero, A., et al., 2010, arXiv:1012.4351

Kaufer, A., Stahl, O., Tubbesing, S., et al., 1999, The Messenger, 95, 8

Paredes, J. M., Martí, J., \& Ribó, M., Massi M. 2000, Science, 288, 2340

Sarty, G. E., Szalai, T., Kiss, L. L., et al., 2011, MNRAS, 411, 1293 (S11)

Wilson, R. E. \& van Hamme, W. 2003, Computing Binary Stars Observables, Ver. 4. 